University of Massachusetts Amherst

ScholarWorks@UMass Amherst

Chemistry Department Faculty Publication Series

Chemistry

1998

\title{
Effect of sample volume on the limit of detection in flow injection hydride generation electrothermal atomic absorption spectrometry
}

JF Tyson

RI Ellis

SA McIntosh

CP Hanna

Follow this and additional works at: https://scholarworks.umass.edu/chem_faculty_pubs

Part of the Chemistry Commons

\section{Recommended Citation}

Tyson, JF; Ellis, RI; McIntosh, SA; and Hanna, CP, "Effect of sample volume on the limit of detection in flow injection hydride generation electrothermal atomic absorption spectrometry" (1998). Journal of Analytical Atomic Spectrometry. 1068.

Retrieved from https://scholarworks.umass.edu/chem_faculty_pubs/1068 


\title{
Effect of sample volume on the limit of detection in flow injection hydride generation electrothermal atomic absorption spectrometry
}

\author{
Julian F. Tyson*a ${ }^{* a}$ Robert I. Ellis ${ }^{a}$, Susan A. McIntosh ${ }^{b}$ and Christopher P. Hanna ${ }^{b}$ \\ ${ }^{a}$ Department of Chemistry, University of Massachusetts, Box 34510, Amherst, MA 01003-4510, USA \\ ${ }^{b}$ Perkin-Elmer Corporation, 761 Main Road, Norwalk, CT06859-0215, USA
}

\begin{abstract}
The analytical performance of methods for the determination of hydride forming elements has been improved recently by the development of procedures in which the hydride is trapped on the interior surface of a graphite furnace atomizer. The signal for a given concentration increases with increase in sample volume and it is often implied that a decrease in the limit of detection may also be achieved by increasing the sample volume. To evaluate this claim, a simple equation was derived which predicts the relationship between detection limit and sample volume when all the contributions to the blank are proportional to sample volume. A time-based approach to the variation of sample volume was developed to ensure that the analyte introduced from reagent contamination was, in fact, proportional to sample volume. Detection limits were measured for a series of sample volumes between 156 and $1560 \mu \mathrm{l}$. As the sample volume was increased, the detection limit improved significantly from 0.3 to around $0.05 \mu \mathrm{g} \mathrm{I}^{-1}$ up to a volume of about $500 \mu \mathrm{l}$. Between 500 and $1000 \mu \mathrm{l}$, a further improvement, to around $0.02 \mu \mathrm{g} \mathrm{I}^{-1}$, was obtained, but for volumes larger than $1000 \mu \mathrm{l}$ no further significant improvement was obtained. Good agreement between the predicted and experimentally determined variations in detection limit with sample volume was obtained and thus the underlying inverse proportionality of the relationship between detection limit and sample volume was confirmed. This rectangular hyperbolic relationship has practical consequences for the extent to which detection limits can be improved by increasing the sample volume, even when the blank is very low or zero.
\end{abstract}

Keywords: Flow injection; hydride generation; electrothermal atomic absorption spectrometry; arsenic; limit of detection; sample volume

The analytical performance of methods for the determination of hydride forming elements has been improved recently by the development of procedures in which the hydride is trapped on the interior surface of a graphite furnace atomizer. ${ }^{1}$ These procedures have the advantages that atomization is independent of (a) minor changes to the surface of the atomizer and (b) the composition of the gaseous medium used to transport the hydride to the atomizer. For the commonly used quartz tube atomizer, both of these factors are relevant and lead to poor day-to-day reproducibility in sensitivity and the need for tedious and time-consuming reconditioning of the atomizer interior surface. In addition, a sample volume much larger than the $20 \mu 1$ typically employed in electrothermal atomic absorption spectrometry (ETAAS) may be used. For example, in one of the first reports of this procedure, ${ }^{2}$ samples between 50 and $1000 \mathrm{ml}$ were used in a batch procedure. This favorable sample volume should give rise to improved sensitivity (as measured by the slope of the calibration) assuming that the generation, separation, transport and trapping processes have high efficiency and should lead to an improved detection limit compared with that obtained for the introduction of a small volume of solution directly into the furnace. The advantages of the hydride generation procedure in terms of separating the analyte from the undesirable matrix components are well documented, as are the various interference effects. ${ }^{3}$

Although the in-atomizer trapping procedure will overcome a number of the vapor phase interferences encountered with the quartz tube atomizer such as scavenging of hydrogen radicals by non-analyte hydrides, interferences in the vapor generation processes (such as those caused by transition metals) remain. These interferences may be diminished by exploiting the kinetic discrimination afforded by hydride generation (HG) in a flow injection (FI) system, a feature which is also well documented. ${ }^{4}$ As the use of flow injection also brings a number of other advantages, such as the automation of the entire procedure, it is likely that FI-HG-AAS will become more widely used.

As one of the advantages of the procedure using a graphite furnace atomizer over the use of the quartz tube atomizer is the improved detection limit, it is relevant to examine the parameters governing this figure of merit. Although many studies have made reference to detection limit, many optimizations of relevant experimental parameters have been carried out with reference to maximizing the sensitivity or some subjective function of sensitivity and throughput.

Sample volume is clearly an important parameter. For both batch $^{2,5,6}$ and flow ${ }^{1,7-17}$ procedures it has been reported that the limit of detection may be improved by increasing the sample volume. In a few papers, data are included in support of this statement, but in many papers the authors simply speculate that this improvement would occur. The impression is often given in discussions of this relationship that (a) detection limit is linearly related to sample volume (as values are given for only two volumes $)^{2,17}$ and (b) the detection limit achievable may be as low as desired-it is simply a matter of making the sample volume as large as necessary. The first of these statements is not true. The relationship between detection limit and sample volume is one of inverse proportion $^{15}$ and an inversely proportional relationship is not linear, although there may be regions in which the relationship approximates to linear. The function relating the two variables is one half of a rectangular hyperbola (that is, a hyperbola whose asymptotes are at right-angles to each other). The second of the statements is only true when there is a contribution to the measured signal from analyte present in the reagents which is either zero or independent of sample volume used. In FI-HG-ETAAS, the signal due to contamination of the reagents by the analyte in fact increases as the sample volume increases, because the quartz probe, which delivers the hydride to the furnace, needs to be positioned inside the furnace for a longer period of time. 


\section{THEORY}

For many trace element determinations, the general relationship between the standard deviation in the concentration domain, $s_{\mathrm{C}}$, and the analyte concentration, $C$ (made up of the concentration in the sample and the concentration added due to contamination of reagents, carryover from previous samples, etc.), may be modelled as a simple linear function ${ }^{18}$

$$
s_{\mathrm{C}}=s_{0}+k C
$$

where $s_{0}$ is the standard deviation of the field blank (a sample with zero analyte concentration) and $k$ is a constant. For ETAAS, the signal ( $A$, peak area in absorbance seconds) is directly related to the mass of the analyte, $m$, and thus if the sample volume is $V$ then

$$
A=S m=S V C
$$

where $S$ is the sensitivity of the method. From Eqn. (2) it may be deduced that the standard deviation in the signal domain, $s_{\mathrm{A}, \mathrm{C}}$ is related to the standard deviation in the concentration domain by

$$
s_{\mathrm{A}, \mathrm{C}}=S V s_{\mathrm{C}}
$$

and thus, for the signal domain, Eqn. (1) becomes

$$
s_{\mathrm{A}, \mathrm{C}}=s_{\mathrm{A}, 0}+S V k C
$$

where $s_{\mathrm{A}, 0}$ is the standard deviation of the signal for the field blank. If the amount of analyte added to the sample from the reagents is directly proportional to sample volume (as would be the case in FI-HG-ETAAS), then if $C_{\mathrm{b}}$ is the concentration of analyte added to the sample from the reagents needed to process a sample volume of $V$, then

$$
s_{\mathrm{A}, \mathrm{C}_{b}}=s_{\mathrm{A}, 0}+S k V C_{\mathrm{b}}
$$

If $C_{\mathrm{s}}$ is the concentration of analyte in the sample, the relationship between peak area, $A$, and analyte concentration [Eqn. (2)] now becomes

$$
A=S V C_{\mathrm{s}}+S V C_{\mathrm{b}}
$$

The signal corresponding to the detection limit, $C_{\mathrm{d} 1}$ would be the intercept on the signal axis, $S V C_{\mathrm{b}}$, plus three times the standard deviation of the signal for the field blank, $3 s_{\mathrm{A}, \mathrm{C}_{b}}$. Substituting this signal from Eqn. (5) into Eqn (6) gives

$$
3 s_{\mathrm{A}, 0}+3 S k V C_{\mathrm{b}}+S V C_{\mathrm{b}}=S V C_{\mathrm{d} 1}+S V C_{\mathrm{b}}
$$

which may be rearranged to give

$$
C_{\mathrm{d} 1}=3 s_{\mathrm{A}, 0} / S V+3 k C_{\mathrm{b}}
$$

From Eqn. (8), it may be seen that as $V$ increases, the detection limit asymptotically approaches the value of $3 k C_{\mathrm{b}}$. Values of $k$ between 0.01 and 0.1 are typical for trace analytical procedures. ${ }^{18}$ On the basis of this simple treatment, the infinitevolume detection limit would be $3 k C_{\mathrm{b}}$, and it would be predicted that the detection limit will improve with increasing sample volume up to this limiting value.

If the blank were independent of sample volume, as might be the case for a batch procedure in which fixed amounts of reagents were used regardless of sample volume, then Eqns. (5) and (6) would be modified to

$$
\begin{gathered}
s_{\mathrm{A}, \mathrm{C}_{b}}=s_{\mathrm{A}, 0}+S k C_{\mathrm{b}} \\
A=S V C_{\mathrm{s}}+S C_{\mathrm{b}}
\end{gathered}
$$

and Eqn. (7) becomes

$$
3 s_{\mathrm{A}, 0}+3 S k C_{\mathrm{b}}+S C_{\mathrm{b}}=S V C_{\mathrm{d} 1}+S C_{\mathrm{b}}
$$

from which

$$
C_{\mathrm{d} 1}=\left[\left(3 s_{\mathrm{A}, 0} / S\right)+3 k C_{\mathrm{b}}\right] / V
$$

and therefore, as all the terms within the square brackets are constants, the detection limit is inversely proportional to sample volume.

In deriving Eqns. (8) and (12), it has been assumed that the process of subtracting the blank introduces no additional variation in the signal. It would be possible to account for this additional source of uncertainty in the above treatment, although this would require an assumption about the nature of the distribution of signals at low analyte concentrations. In general, this distribution is non-Gaussian. ${ }^{19}$ However, for the purposes of estimating the effect of the propagation of error, if a Gaussian distribution is assumed, the effect of including this additional source of uncertainty is to introduce $\sqrt{2}$ into each term on the right-hand side of Eqn. (8), which now becomes

$$
C_{\mathrm{dl}}=3 \sqrt{2} s_{\mathrm{A}, 0} / S V+3 \sqrt{2} k C_{\mathrm{b}}
$$

In this paper, we examine the variation in measured concentration detection limit as a function of sample volume for the situation in which the contribution from the blank is proportional to sample volume and compare the results obtained with the predictions of Eqns. (8) and (13). The implications for the design of procedures with improved detection limits are discussed.

\section{EXPERIMENTAL}

\section{Instrumentation}

The flow injection manifold, shown in Fig. 1, was configured with a Perkin-Elmer (Norwalk, CT, USA) FIAS 200 unit connected to a Perkin-Elmer 4100ZL Zeeman corrected electrothermal atomic absorption spectrometer interfaced with a Digital 316sx workstation, and controlled using Perkin-Elmer Gem software (version 7.2.1). The manifold was constructed using PTFE manifold tubing and Perkin-Elmer Chemifold flow injection fittings. The gas-liquid separator was a plastic Perkin-Elmer FIAS device ( part number B050-7959). A Perma Pure Nafion ${ }^{\circledR}$ dryer (Model MD-110.12F) was fitted to the gas transfer line through which the hydrides were transported to the electrothermal atomizer, to remove moisture from the transferred gases. ${ }^{20}$ The argon flow rate was $130 \mathrm{ml} \mathrm{min}^{-1}$. The arsine was trapped on a transversely heated graphite tube pre-heated with $120 \mu \mathrm{l}$ of $0.1 \% \mathrm{~m} / \mathrm{v}$, iridium chloride solution (Perkin-Elmer). ${ }^{21}$ Tubes which are pre-treated with iridium may be used for up to 300 firings provided the temperature does not exceed $2300^{\circ} \mathrm{C}^{22}$ A Perkin-Elmer System II electrodeless discharge lamp operated at $260 \mathrm{~mA}$ was used with detection at $193.7 \mathrm{~nm}$. Peak area data were used for all determinations. The furnace programme used is shown in Table 1 .

\section{Reagents}

A stock standard arsenic solution was prepared by diluting an aliquot of $\mathrm{As}^{\mathrm{V}}$ atomic standard solution (Perkin-Elmer) to

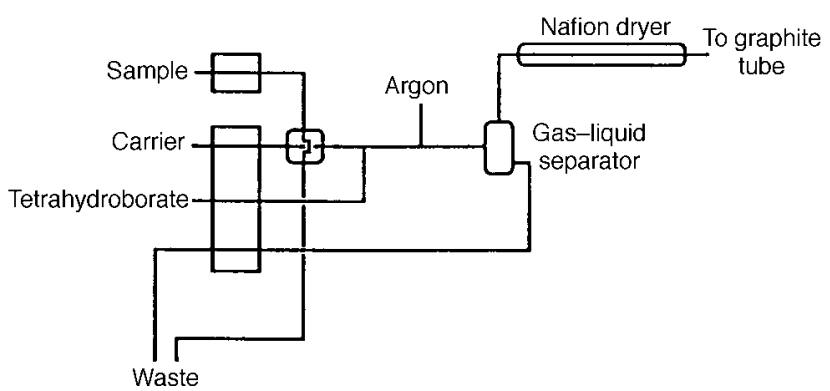

Fig. 1 Flow injection manifold. The flow rate of the hydrochloric acid carrier solution was $5.6 \mathrm{ml} \mathrm{min} \mathrm{min}^{-1}$ and that of the sodium tetrahydroborate solution was $3.6 \mathrm{ml} \mathrm{min}^{-1}$. 
Table 1 Furnace program

\begin{tabular}{ccccc} 
Step & Temperature $/{ }^{\circ} \mathrm{C}$ & Ramp/s & Hold $/ \mathrm{s}$ & $\mathrm{Read} / \mathrm{s}$ \\
1 & 400 & 1 & 15 & \\
2 & 2100 & 0 & 5 & 5 \\
3 & 2300 & 1 & 3 & \\
\hline
\end{tabular}

produce a solution of $1000 \mu \mathrm{g} 1^{-1}$. The arsenic atomic standard solution is made using $\mathrm{As}^{\mathrm{v}}$ because this oxidation state is the most stable state for storage. Arsine is more easily formed from the $A s^{\text {III }}$ oxidation state, so pre-reduction of arsenic in the samples is necessary. The carrier stream was $10 \% \mathrm{v} / \mathrm{v}$ hydrochloric acid (Fisher, Pittsburgh, PA, USA) and the reductant stream was $0.2 \% \mathrm{~m} / \mathrm{v} \mathrm{NaBH}$ (Fisher) prepared daily and stabilized with $0.05 \% \mathrm{~m} / \mathrm{v} \mathrm{NaOH}$ (Fisher). Samples were pre-reduced by adding $10 \mathrm{ml}$ of concentrated $\mathrm{HCl}, 10 \mathrm{ml}$ of a $10 \% \mathrm{~m} / \mathrm{v} \mathrm{KI}$ (Fisher) solution and $10 \mathrm{ml}$ of a $10 \% \mathrm{~m} / \mathrm{v}$ ascorbic acid (Fisher) solution to a $100 \mathrm{ml}$ calibrated flask, followed by addition of an aliquot of arsenic stock solution. These solutions were stoppered and left at room temperature for $1 \mathrm{~h}$ to allow reduction of $\mathrm{As}^{\mathrm{V}}$ to $\mathrm{As}^{\mathrm{III}}$ prior to dilution to volume.

\section{Procedure}

In the normal mode of operation of this technique, the loop of the flow injection valve is filled with sample solution and the quartz capillary probe, which delivers the gases from the gas-liquid separator, is inserted into the graphite tube. The valve is then switched to the inject position and reagents are pumped for a period long enough to allow the sample to pass completely through the manifold. In this process the acidified sample reacts on-line with the sodium tetrahydroborate forming arsine. Following the addition of argon stripping gas, the volatile arsine reaches the gas-liquid separator from which it is swept through the gas transfer line to the graphite tube. The quartz probe is removed before firing the furnace. Zeeman effect background correction is not required since matrix removal occurs in the manifold.

The rate of hydride transport will be low for an initial period while the sample is carried to the gas-liquid separator, then it will rise sharply and reach a maximum before declining as the sample is flushed through the manifold.

Whenever the gas-liquid separator and the graphite tube are connected, arsine from the reagents will be collected. The time required to flush a given sample volume through the gasliquid separator is not directly proportional to the sample loop volume because of the dispersion on the leading and training edges. Therefore, the blank would not vary in proportion to the sample volume but would increase relative to the analyte signal.

This problem was avoided by the use of a time-based approach for which the blank accumulation was proportional to sample volume. A sample loop of $2000 \mu$ l (in excess of the required sample volume) was fitted to the valve. The loop was filled, the valve switched to the inject position and sample passed through the manifold until the rate of transfer of arsine from the gas-liquid separator had reached a steady maximum. The quartz probe was then inserted into the furnace. The probe remained in place for a defined time, during which a sub-sample of the total arsine generated was delivered to the furnace. The probe was then removed. The sample volume was calculated as the volume pumped in the sample line during the time that the probe was in the furnace. The arsine was thus transferred to the atomizer at a steady maximum rate, and the contribution to the total signal from analyte in the reagents was in direct proportion to the sample volume.

The flow rate in the sample line was measured by first filling

\section{Table 2 Flow injection program}

$\begin{array}{ccccc}\text { Step } & \text { Time/s* } & \text { Pump 1/rpm } & \text { Pump 2/rpm } & \begin{array}{c}\text { Valve } \\ \text { position }\end{array} \\ \text { Pre-fill } & 5 & 100 & 0 & \text { Fill } \\ 1 & 45 & 100 & 80 & \text { Fill } \\ 2 & 7 & 0 & 80 & \text { Inject } \\ 3 & 8 & 0 & 0 & \text { Inject } \\ 4 & {\mathrm{C}^{\dagger}}^{\dagger} & 0 & 80 & \text { Inject } \\ 5 & 8 & 0 & 0 & \text { Inject } \\ 6 & 5 & 0 & 80 & \text { Fill }\end{array}$

* An entry of 8 in the time column causes the system to insert/ remove the probe from the furnace. ${ }^{\dagger}$ Time corresponding to sample volume.

the manifold with water, then pumping a dilute Methyl Red solution with a small air bubble between the water and the colored solution. The air bubble prevented dispersion at the front of the colored solution as it traveled through the manifold. The rate of flow through the $2000 \mu \mathrm{l}$ sample loop was determined with the aid of a stop-watch. Hence the sample volume could be defined by the sample injection time. The flow injection program is shown in Table 2 .

Standards containing $0.2 \mu \mathrm{g} 1^{-1}$ arsenic were measured 10 times and solutions containing 2,5 and $10 \mu \mathrm{g} 1^{-1}$ arsenic were measured three times. The analysis of these samples was performed using 10 different deposition times between 2 and $20 \mathrm{~s}$, corresponding to $10 \mathrm{sub}$-sample volumes ranging between 156 and $1560 \mu \mathrm{l}$. The linear regression equation for peak area as a function of concentration was calculated for each subsample volume. The slopes were plotted as a function of sample volume from which a value of $S$ was obtained, from which the characteristic mass was calculated. The mean and standard deviation of the signals for the $0.2 \mu \mathrm{g}^{-1}$ samples were calculated. The limits of detection, calculated as the concentration derived from the regression equation corresponding to the intercept plus three times the standard deviation of the 10 replicates of the $0.2 \mu \mathrm{g}^{-1}$ solution were calculated. An estimate of the blank concentration, $C_{\mathrm{b}}$, was made from the intercepts on the concentration axis of the calibration plots. A value for $s_{\mathrm{A}, 0}$ [see Eqn. (4)] was obtained from a plot of the standard deviations of the response to the $0.2 \mu \mathrm{g} 1^{-1}$ solution against the total mass of analyte (that due to the sub-sample and that due to the blank). From the slope of this plot [equal to $S k$, see Eqn. (4)] and the value of $S$ already determined, a value of $k$ was calculated.

Some further manipulations of the data were performed. A value of the detection limit at infinite volume was estimated from the plot of concentration detection limit as a function of sample volume, from which a further estimate of $k$ [see Eqn. (1)] was made. The slope and intercept of an unweighted linear regression plot of concentration detection limit against the reciprocal volume were calculated, together with the corresponding $95 \%$ confidence intervals.

\section{RESULTS AND DISCUSSION}

The slope and intercepts (on both axes) of the calibration plots at the 10 different sample volumes are given in Table 3 . The slope of the calibration increased linearly with increase in subsample volume up to $1092 \mu$ l, beyond which curvature set in. The equation for the unweighted linear least-squares regression line, omitting the last two points, was slope of calibration $=$ $1.023 \times 10^{-4}$ (sample volume in $\left.\mu \mathrm{l}\right)+5.36 \times 10^{-4}$, with correlation coefficient of 0.998 . Hence the sensitivity, $S$, is $1.023 \times 10^{-4}$ absorbance $\mathrm{sg}^{-1}$ and the characteristic mass is $43 \mathrm{pg}$. The latter compares favorably with the manufacturer's value of $40 \pm 8 \mathrm{pg}$. As the sub-sample volume increased, the 
Table 3 Calibration data for different sample volumes

\begin{tabular}{|c|c|c|c|}
\hline $\begin{array}{l}\text { Sample } \\
\text { volume/ } \mu 1\end{array}$ & 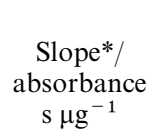 & $\begin{array}{c}\text { Intercept* } \\
\text { on response } \\
\text { axis/absorbance } \\
\text { s }\end{array}$ & $\begin{array}{l}\text { Intercept on } \\
\text { concentration } \\
\text { axis } / \mu \mathrm{g} \mathrm{l}^{-1}\end{array}$ \\
\hline 156 & 0.016 & 0.014 & 0.875 \\
\hline 312 & 0.033 & 0.019 & 0.576 \\
\hline 468 & 0.048 & 0.026 & 0.542 \\
\hline 624 & 0.063 & 0.033 & 0.524 \\
\hline 780 & 0.080 & 0.036 & 0.450 \\
\hline 936 & 0.100 & 0.035 & 0.350 \\
\hline 1092 & 0.114 & 0.040 & 0.351 \\
\hline 1248 & 0.125 & 0.045 & 0.360 \\
\hline 1404 & 0.132 & 0.049 & 0.371 \\
\hline 1560 & 0.136 & 0.054 & 0.397 \\
\hline
\end{tabular}

uncertainty in the slope of the calibration increased. The signal for the $10 \mu \mathrm{g} 1^{-1}$ samples with the two largest sample volumes (1404 and $1560 \mu \mathrm{l})$ was a flat-topped peak, as the central part of the injected sample was not diluted by the carrier stream. Thus as the sample volume is increased, the range of concentrations in the linear calibration may be reduced.

The blank concentration, calculated as the average of the intercepts for the calibrations on the concentration axis (excluding the calibration for $156 \mu$, rejected on the basis of a $Q$ test), was $0.44 \mu \mathrm{g} 1^{-1}$ with a standard deviation $(n=9)$ of $0.09 \mu \mathrm{g} 1^{-1}$. The means of the responses for the $0.2 \mu \mathrm{g}^{-1}$ standard $(n=$ 10) for the various sample volumes are given in Table 4, together with the corresponding standard deviations. Also given in Table 4 is the total analyte mass (given by the product of the sample volume and the sum of the sample concentration, $0.2 \mu \mathrm{g}^{-1}$, and the blank concentration, $0.44 \mu \mathrm{g}^{-1}$ ). A plot of standard deviation against sample mass had an unweighted linear least-squares regression equation of standard deviation of signal $=5.03 \times 10^{-7}$ (sample mass) $+1.01 \times 10^{-3}$. Thus at zero analyte mass, the standard deviation is $1.01 \times 10^{-3}$. This is an estimate of $s_{\mathrm{A}, 0}$ [see Eqn. (4)].

The variation of concentration detection limit with sample volume is shown in Fig. 2. It can be seen that the general trend predicted by Eqn. (8) is obtained, namely as the volume increases the detection limit decreases and approaches a limiting (infinite volume) value. However, closer inspection reveals that around $1000 \mu \mathrm{l}$ there is an apparent increase in the concentration detection limit, suggesting that the simple model may no longer be valid. It can be seen from the overlap of the estimated $95 \%$ confidence intervals for adjacent points that the effect may not be significant. Extrapolation of the curve

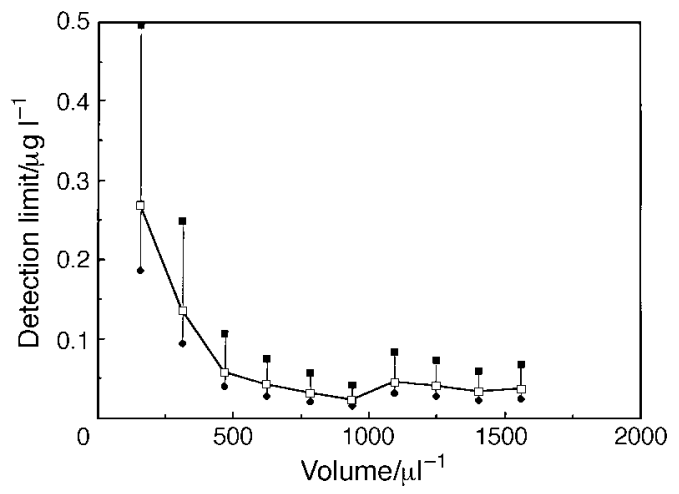

Fig. 2 Variation of limit of detection $\left(\mu \mathrm{g} 1^{-1}\right)$ with sample volume $(\mu \mathrm{l})$.

gives a value of about $0.03 \mu \mathrm{g} 1^{-1}$ for the infinite volume detection limit. From this and the value of $0.44 \mu \mathrm{g} 1^{-1}$ for $C_{\mathrm{b}}$, a value of $k$ [see Eqn. (1)] of 0.023 is calculated, which is within the range considered typical for instrumental methods. ${ }^{18}$

An alternative method for estimating $k$ is to use the slope of the plot of standard deviation of the signal versus total mass, $5.03 \times 10^{-7}$, which from Eqn. (4) is equal to $S k$. Using the value of $S$ of $1.023 \times 10^{-4}, k$ is calculated to be 0.004 . This suggests that the value of the extrapolated infinite volume detection limit is too high.

A plot of $C_{\mathrm{d} 1}$ versus $1 / V$ is given in Fig. 3. Visual inspection of the data shows a group of four data points which may possibly deviate from the linear relationship formed by the remaining six points. As was pointed out for the data displayed in Fig. 2, the overlap of the $95 \%$ confidence intervals for

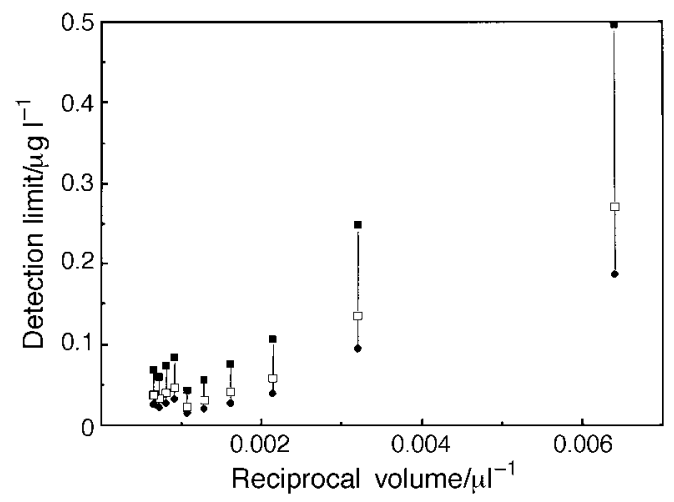

Fig. 3 Scatter plot of detection limit $\left(\mu \mathrm{g}^{-1}\right)$ as a function of the reciprocal sample volume $\left(\mu 1^{-1}\right)$.

Table 4 Signal characteristics for $0.2 \mu \mathrm{g} 1^{-1}$ standard, total mass of analyte and detection limit for different sample volumes

\begin{tabular}{|c|c|c|c|c|c|}
\hline $\begin{array}{c}\text { Sample } \\
\text { volume } / \mu l\end{array}$ & $\begin{array}{c}\text { Mean } \\
\text { response/ } \\
\text { absorbance s }\end{array}$ & $\begin{array}{c}\text { Standard } \\
\text { deviation/ } \\
\text { absorbance s }\end{array}$ & Analyte mass/pg & 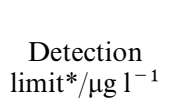 & $\begin{array}{l}95 \% \text { confidence interval } \\
\text { about detection limit }\end{array}$ \\
\hline 156 & 0.01793 & 0.00144 & 99.8 & 0.271 & $0.187-0.496$ \\
\hline 312 & 0.02687 & 0.00150 & 200 & 0.136 & $0.094-0.249$ \\
\hline 468 & 0.03570 & 0.00092 & 300 & 0.058 & $0.040-0.106$ \\
\hline 624 & 0.04597 & 0.00087 & 399 & 0.041 & $0.028-0.075$ \\
\hline 780 & 0.05245 & 0.00082 & 499 & 0.031 & $0.021-0.057$ \\
\hline 936 & 0.06116 & 0.00077 & 599 & 0.023 & $0.016-0.042$ \\
\hline 1092 & 0.07017 & 0.00177 & 699 & 0.046 & $0.032-0.084$ \\
\hline 1248 & 0.07625 & 0.00167 & 799 & 0.040 & $0.028-0.073$ \\
\hline 1404 & 0.08116 & 0.00146 & 899 & 0.033 & $0.023-0.060$ \\
\hline 1560 & 0.08557 & 0.00168 & 998 & 0.037 & $0.025-0.068$ \\
\hline
\end{tabular}

* Only one figure would be significant; the others are given for information. ${ }^{\dagger}$ Values calculated from the $95 \%$ confidence interval about the standard deviation of each set of 10 replicate measurements (the interval is not symmetric-see ref. 23, p. 38). As no account is taken of the confidence interval about the slope of the line, these values will underestimate the confidence interval about the detection limit. For a fuller discussion of the estimation of this interval, see ref. 23 , p. 266. 
adjacent points may mean that the differences are not significant. The least-squares regression line of these six points has a slope of 41.0 and an intercept of $7.19 \times 10^{-3}$ with a correlation coefficient of 0.999 . From this value of the intercept, $k$ is calculated to be 0.005 [Eqn. (8)] or 0.004 [Eqn. (13)], in agreement with the value calculated above.

The slope of the plot may be compared with the values calculated from Eqn. (8) (29.4) and Eqn. (13) (41.6), from which it may be seen that Eqn. (13) gives much better agreement with the experimentally determined slope. If all 10 points are included in the plot, the slope becomes 42.3 and the intercept becomes $-7.92 \times 10^{-3}$. The $95 \%$ confidence values about these values are \pm 7.4 and \pm 0.018 for the slope and intercept, respectively.

\section{CONCLUSIONS}

The agreement between the predicted values of the slope and intercept of the plot of $C_{\mathrm{d} 1}$ versus $1 / V$ suggest that Eqn. (13) is a reasonable model for the situation encountered in FI-HG-ETAAS. Therefore, in order to obtain the lowest possible detection limit, it is necessary to reduce as far as possible the contribution to the analyte signal from the reagents used. Instrumental modifications which decreased the characteristic mass would also be beneficial, as would control over the features which influence the signal variation in the absence of analyte. For absorption spectrometric techniques these latter features are well known, and discussions may be found in standard texts (e.g., ref. 24). Even for the experimental situation used in these studies, it is likely that there are contributions to the blank signal from sources other than reagent contamination, such as carryover from one sample to the next. It is possible, therefore, that the blank is made up of contributions which are dependent on sample volume and contributions which are independent of sample volume. For the latter situation, an equation analogous to Eqn. (13) may also be derived on the basis of a Gaussian distribution of errors, but gives a much more complicated equation which shows a minimum in the relationship between detection limit and sample volume. It is possible that the data obtained in the experiments reported here support this more complex model. It may be concluded that for the experiments reported here, there is little point in using sample volumes in excess of $1000 \mu \mathrm{l}$ and that a considerable increase in throughput could be obtained with little effect on detection limit by using a $500 \mu \mathrm{l}$ sample volume. Even if the blank is constant (or zero), the nature of the rectangular hyperbola relationship needs to be kept in mind. As the sample volume is increased, there will eventually come a point beyond which the improvement in detection limit obtainable is not significant or is made only with considerable costs in terms of throughput. Previously reported data which suggest that the detection limit improves linearly with sample volume are presumably taken from the initial 'steep' decrease of the rectangular hyperbola.

Financial support for Robert I. Ellis by Perkin-Elmer is gratefully acknowledged.

\section{REFERENCES}

1 Matusiewicz, H., and Sturgeon, R. E., Spectrochim. Acta, Part B, 1996, 51, 377.

2 Lee, D. S., Anal. Chem., 1982, 54, 1682.

3 Dèdina, J., and Tsalev, D. L., Hydride Generation Atomic Absorption Spectrometry, Wiley, Chichester, 1995.

4 Fang, Z., Flow Injection Atomic Absorption Spectrometry, Wiley, Chichester, 1995.

5 Thompson, K. C., and Thomerson, D. R., Analyst, 1974, 99, 595.

6 Sturgeon, R. E., Willie, S. N., Sproule, G. I., Robinson, P. T., and Berman, S. S., Spectrochim. Acta, Part B, 1989, 44, 667.

7 Baxter, D. C., and Frech, W., Anal. Chim. Acta, 1989, 225, 175.

8 Veber, M., Čujes, K., and Gomišcek, S., J. Anal. At. Spectrom., 1994, 9, 285.

9 Zhang, L., McIntosh, S., Carnrick, G. R., and Slavin, W., Spectrochim. Acta, Part B, 1992, 47, 701.

10 Doidge, P. S., Sturman, B. T., and Rettburg, T. M., J. Anal. At. Spectrom., 1989, 4, 251.

11 Yan, X.-P., and Ni, Z.-M., J. Anal. At. Spectrom., 1991, 6, 483.

12 Zhang, L., Ni, Z.-M., and Shan, X.-Q., Spectrochim. Acta Part B, 1989, 44, 339 .

13 Erber, D., Bettmer, J., and Camman, K., Fresenius' Z. Anal. Chem., 1994, 349, 738.

14 Matusiewicz, H., Suzka, A., and Kopraz, M., Microchem. J., 1995, 52, 286.

15 Sinemus, H. W., Stabel, H. H., Raziuk, B., and Kleiner, J., Spectrochim. Acta, Part B, 1993, 48, 643.

16 Sturgeon, R. E., Willie, S. N., and Berman, S. S., J. Anal. At. Spectrom., 1989, 4, 443.

17 Infante, H. G., Fernandez-Sanchez, M. L., and Sanz-Medel, A., J. Anal. At. Spectrom., 1996, 11, 571 .

18 Analytical Methods Committee, Analyst, 1987, 112, 199.

19 Eckschlager, K., Errors, Measurements and Results in Chemical Analysis, Van Nostrand Reinhold, London, 1969, p. 103.

20 Sundin, N. G., Tyson, J. F., McIntosh, S. A., and Hanna, C. P., Spectrochim. Acta, Part B, 1995, 50, 369.

21 Perkin-Elmer Manufacturer's Instruction Manual, part number B050-9907, Publication B3212.10, Perkin-Elmer, Norwalk, CT, 1993.

22 Hanna, C. P., Carnrick, G. R., McIntosh, S., Guyette, L. C., and Bergemann, D. E., At. Spectrosc., 1995, 16, 82.

23 Liteanu, C., and Rica, I., Statistical Theory and Methodology of Trace Analysis, Ellis Horwood, Chichester, 1980.

24 Ingle, J. D., and Crouch, S. R., Spectrochemical Analysis, PrenticeHall International, London, 1988, p. 150.

Paper 7/04666I

Received July 2, 1997

Accepted October 8, 1997 